\title{
A Bio-inspired Lanthanum-ortho Quinone Catalyst for Aerobic Oxidation of Alcohol
}

\author{
Ruipu Zhang, ${ }^{2,3}$ Long Zhang, ${ }^{1}$ Ming-Tian Zhang, ${ }^{1}$ Sanzhong Luo ${ }^{1,2 *}$
}

${ }^{1}$ Center of Basic Molecular Science, Department of Chemistry, Tsinghua University, Beijing, 100084, China

${ }^{2}$ Key Laboratory for Molecular Recognition and Function, Institute of Chemistry, Chinese Academy of Sciences, Beijing, 100190, China

${ }^{3}$ University of Chinese Academy of Sciences, Beijing, 100049, China

*Correspondence to: $\underline{\text { luosz @ tsinghua.edu.cn. }}$

Abstract. Oxidation reactions are fundamental transformations in organic synthesis and chemical industry. With oxygen or air as terminal oxidant, aerobic oxidation catalysis provides the most sustainable and economic oxidation processes. Most aerobic oxidation catalysis employs redox metal as its active center. While nature provides non-redox metal strategy as in pyrroloquinoline quinone (PQQ)-dependent methanol dehydrogenases (MDH), such an effective chemical version is unknown. Inspired by the recently discovered rare earth metal-dependent enzyme Ln-MDH, here we show that an open-shell semi-quinone anionic radical species in complexing with lanthanum could serve as a very efficient aerobic oxidation catalyst under ambient conditions. In this catalyst, the lanthanum metal serves only as a Lewis acid promoter and the redox process occurs exclusively on the semiquinone ligand. The catalysis is initiated by $1 \mathrm{e}^{-}$-reduction of lanthanum-activated ortho-quinone to a semiquinone-lanthanum complex $\mathrm{La}\left(\mathbf{S Q}^{-*}\right)_{2}$, which undergoes a coupled O-H/C-H dehydrogenation for aerobic oxidation of alcohols with up to 330 $\mathrm{h}^{-1}$ TOF. This study suggests a possible functional mode of semiquinone radicals, widely observed with quinoproteins in Nature. Moreover, this unique reductive activation strategy as well as the 
resulted radical anion as redox ligand creates a new turning point in the development of efficient aerobic oxidation catalysis.

Rare earth metals are in fact not rare but widely distributed in earth crust and lanthanides such as $\mathrm{La}$ and $\mathrm{Ce}$ are as abundant as $\mathrm{Cu}$ and $\mathrm{Zn}^{1}$. In the living sphere, nature has evolved effective strategies in utilizing earth crust metals as active centers in enzymes. However, a rare earth-metal dependent enzyme was not discovered till 2011, when the first lanthanide enzyme, Ln-MDH was discovered in methylotrophic bacterial by Kawai ${ }^{2}$. Ln-MDH is a lanthanide-dependent methanol dehydrogenase $(\mathrm{MDH})^{3}$, closely resembling its calcium counterpart first discovered in $1967^{4}$. Both MDHs contains a redox active cofactor, pyrroloquinoline quinone (PQQ) where the oxidation reaction takes place. Mechanistically, the redox cycle between PQQ and its natural substrate methanol proceeds through either an addition-elimination process with a hemiacetal intermediate or direct hydride transfer (Fig 1a). In both cases, the active sites metals are believed to serve as only non-redox Lewis acid promoter ${ }^{5,6}$. This mechanistic scenario of Ln-MDH is distinctive from the established alcohol oxidation catalysts wherein redox-active metals such as $\mathrm{Pd}^{7-10}, \mathrm{Cu}^{11-14}$, and $\mathrm{Fe}^{15}$ are involved.

The unique mechanistic feature of MDH has drawn significant efforts in pursuing bio-inspired catalysis. Ohshiro demonstrated the synthetic application of PQQ in the oxidation of glucose ${ }^{16}$. Fukuzumi reported a model study of PQQ ester-Ca $\left(\mathrm{ClO}_{4}\right)_{2}$ in the aerobic oxidation of alcohols ${ }^{17,18}$. Schelter synthesized a La-MDH model complex and investigated its catalytic performance in the dehydrogenation of benzyl alcohol with ferricenium ion as an oxidant ${ }^{19}$. In the latter two cases, catalytic turnover was only observed in the presence of strong organic base, but the efficiency was still too low to be of any synthetic utility, particularly when comparing with the redox-metal catalysts such as $\mathrm{Pd}$ or $\mathrm{Cu}$-nitroxyl catalytic system in aerobic oxidation. To achieve effective 
quinone catalysis remains an open challenge from the synthetic point of view, in spite of the ubiquitous existence of quinone-enzyme in nature. Recently, we and others have developed bioinspired ortho-quinone catalysts with the cofactor of copper amine oxidase, $\mathrm{TPQ}^{20}$ as a blueprint for the oxidation of amines ${ }^{21-23}$. In extending to oxidation of alcohols, we discovered that an openshell semi-quinone anionic radical species in complexing with lanthanum served as a highly efficient catalyst for the oxidation of alcohols under aerobic conditions. The catalysis was initialized by $1 \mathrm{e}^{-}$-reduction of lanthanum-activated ortho-quinone $\boldsymbol{o}-\mathbf{Q}$ to a semiquinonelanthanum complex $\mathrm{La}\left(\boldsymbol{o}-\mathbf{Q}^{-}\right)_{2}$, which underwent a concerted dehydrogenation for aerobic oxidation of alcohols with up to $330 \mathrm{~h}^{-1}$ TOF (Fig. 1b).

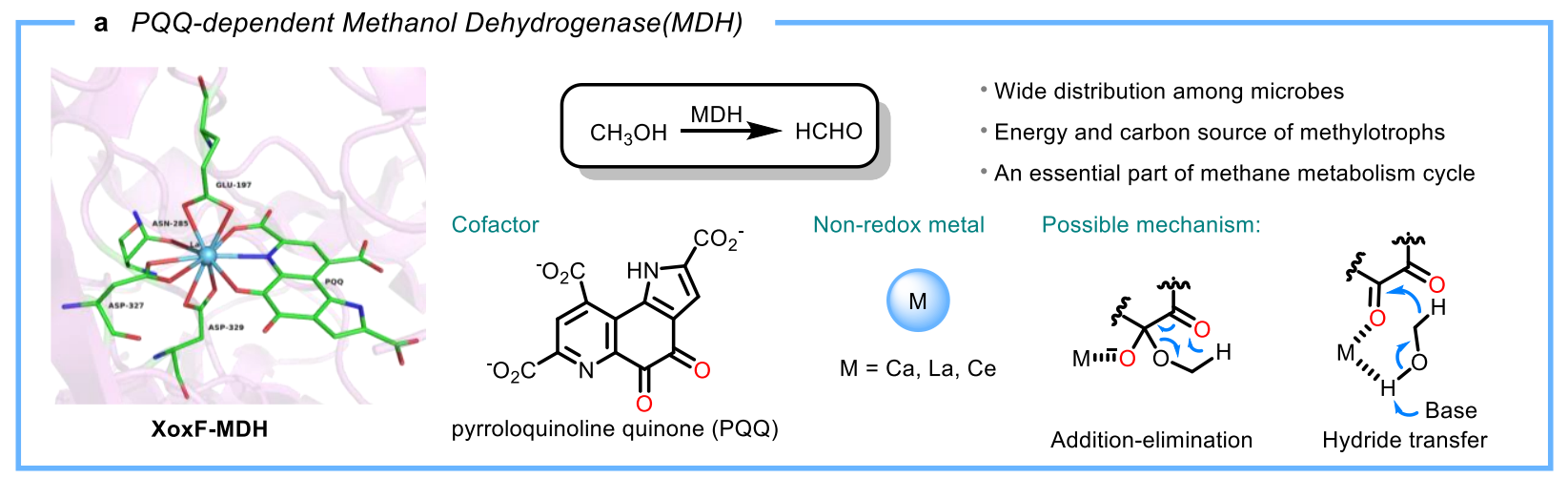

b Bioinspired ortho-Quinone Catalysis

Previous examples

- Ohshiro

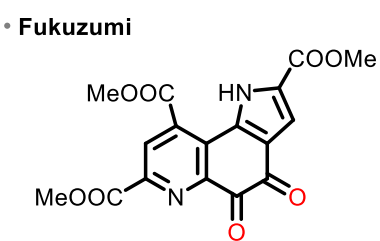

hv or $\mathrm{Ca}^{2+} / \mathrm{DBU}$

Oxidant: $\mathrm{O}_{2}$

$\mathrm{R}_{\mathrm{OH}} \mathrm{TON}=15(65 \mathrm{~h})$
- Schelter

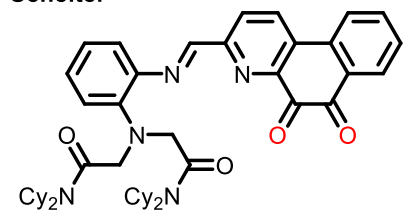

$\mathrm{La}^{3+} / 2,6$-Lutidine Oxidant: $\left[\mathrm{Fc}_{\mathrm{C}}\right]\left[\mathrm{PF}_{6}\right]$

$\mathrm{R}_{\mathrm{OH}} \mathrm{TON}=17(21 \mathrm{~h})$

This work: Lanthanum-ortho-Quinone $\mathrm{H} / \mathrm{H}$ process

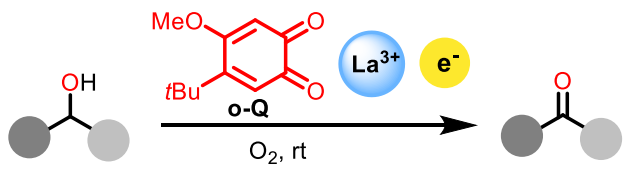

- TOF up to $330 \mathrm{~h}^{-1}$

- Broad substrate scope

- Activation by reduction

- Non-redox metal center

- Concerted H/H transfer with redox ligand only 
Figure 1. Aerobic oxidation of alcohols. (a) Left: active sites of XoxF-methanol dehydrogenase (La-MDH) (PDB ID: 6adm). Right: overview of methanol dehydrogenase. (b) Bio-inspired orthoquinone catalysis. Upper: previous reports about redox properties of PQQ model compounds. Lower: overview of this work.

\section{Results and Discussion}

We initially found that a three-component system composed of $\mathrm{La}(\mathrm{OTf})_{3}$, ortho-quinone $\boldsymbol{o}-\mathbf{Q}$ (for performance of other quinones, see Fig. S1) and $n$-Bu $\mathrm{B}_{4} \mathrm{NI}$ was active for the aerobic oxidation of benzyl alcohol 1a (Fig. 2a). A survey of different metal salts indicated all rare earth elemental (REE) metals worked well in the reactions with lanthanum as the optimal choice in terms of yields (Fig. 2c). In contrast, other metals including base metals such as $\mathrm{Mg}, \mathrm{Li}, \mathrm{Ca}$ or redox metals such as $\mathrm{Cu}, \mathrm{Fe}$ or Pd showed rather poor activity or were even inert (Fig. 2c). We quickly identified $\mathrm{LaI}_{3}$ as the optimal choice in lieu of $\mathrm{La}(\mathrm{OTf})_{3}$ and iodide additive and the two-component catalyst $\mathrm{LaI}_{3} / \boldsymbol{o}-\mathbf{Q}$ was even more active. The reaction reached to completion in 20 mins with only 1 mol \% loading of $\mathrm{LaI}_{3}-(\boldsymbol{o}-\mathbf{Q})_{2}$ (Fig. 2b). The optimal ratio of La/o-Q was determined to be 1: 2 and further increasing the loading of quinone did not lead to any further improvement (Fig. 2b). 

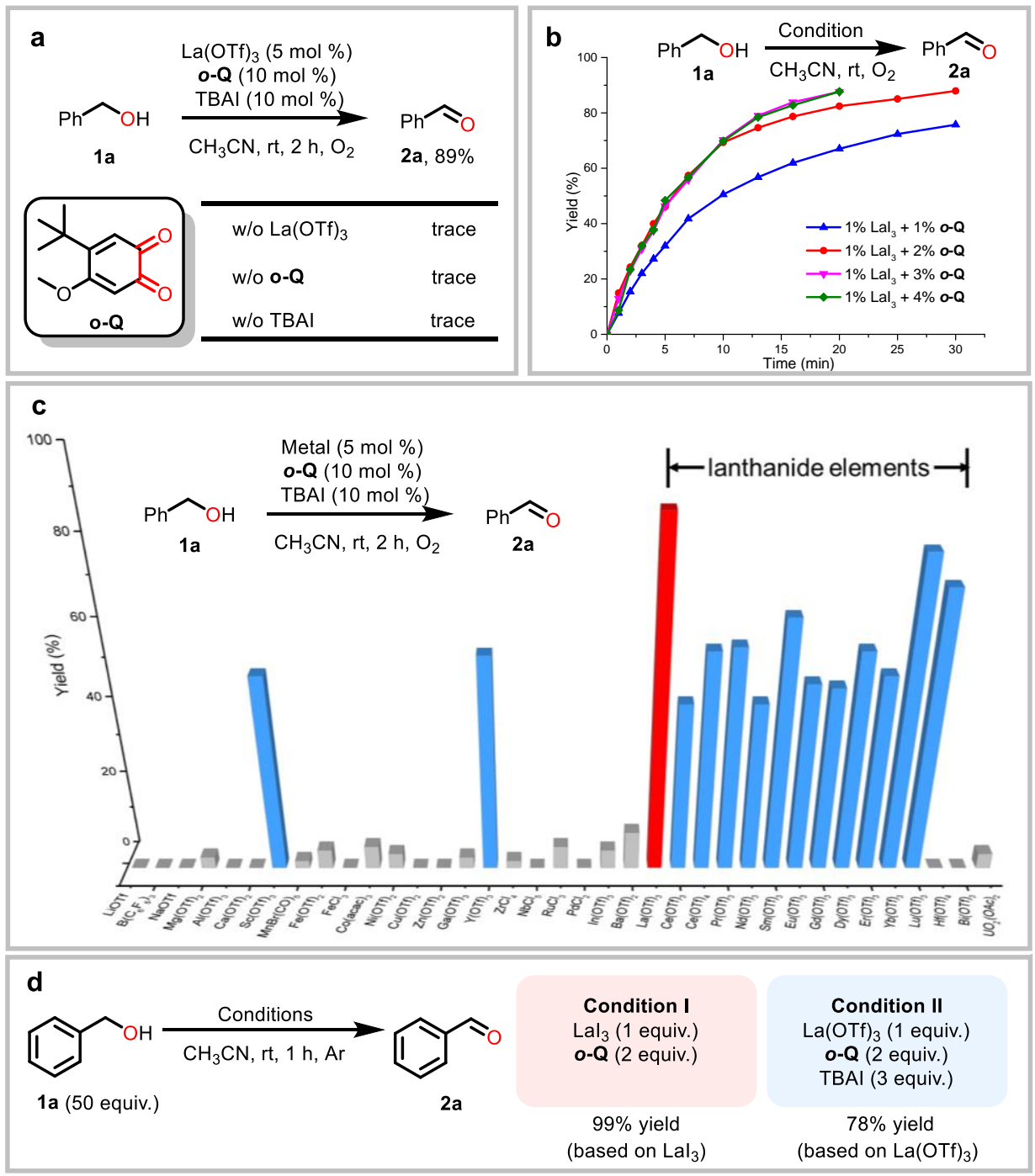

Figure 2. Reaction details. (a) Control experiment. (b) Kinetic profile of the reaction. (c) Metal screening (for details, see Table S1). Unless noted, reactions were conducted on a 0.4 mmol scale with $0.6 \mathrm{~mL} \mathrm{MeCN}$ at room temperature under $1 \mathrm{~atm} \mathrm{O}_{2}$, and yields were determined by $\mathrm{GC}$ using 1,3,5-trimethoxybenzene as internal standard. (d) Stochiometric reactions.

The real active catalytic species was first investigated. In control experiments, removing any of the three catalytic species, $\boldsymbol{o}-\mathbf{Q}, \mathrm{La}(\mathrm{OTf})_{3}$ or TBAI completely shut down the reaction (Fig. 2a), which implied $\mathrm{La}^{3+}$, ortho-quinone catalyst and iodide additive were all essential for this aerobic oxidation. Stoichiometric reactions with either $\mathrm{LaI}_{3}-\boldsymbol{o}-\mathbf{Q}$ or $\mathrm{La}(\mathrm{OTf})_{3}-\boldsymbol{o}-\mathbf{Q}-\mathrm{TBAI}$ proceeded smoothly under argon (Fig. 2d, Condition I and II), suggesting that the substrate-oxidizing active 
specie was generated from $\mathrm{LaI}_{3}$ and $\boldsymbol{o}-\mathbf{Q}$, and oxygen served as the terminal oxidant for the recycling of the catalyst.

Dramatic color change was noted during the reaction process. When iodide additive was added to a solution of $\mathrm{La}(\mathrm{OTf})_{3}$ and $\boldsymbol{o}-\mathbf{Q}$, or upon mixing $\mathrm{LaI}_{3}$ with $\boldsymbol{o}-\mathbf{Q}$, an instant color change from red to dark green was observed (Fig. 3a) and the color changed back to red when the reaction was complete. UV-visible spectrum of the dark green solution indicated a new absorption at 570 and $750 \mathrm{~nm}$. Previously, similar absorptions were reported for semiquinone radical anion in the presence of metal ion such as $\mathrm{Sc}(\mathrm{III})$ or $\mathrm{Zn}(\mathrm{II})^{24,25}$ (Fig. 3a). It should be noted a Ce-MDH was crystallized with their active site existed in the form of a $\mathrm{Ce}(\mathrm{III})$-semiquinone anionic radical complex ${ }^{26}$. However, its mechanistic relevance remains obscure. EPR spectrum of the catalytic system confirmed the existence of an organic radical species with $g=2.003$ (Fig. 3b), and the simulation supported a semiquinone radical in coordination with $\mathrm{La}(\mathrm{III})^{27}$ (Fig. S2). In comparison, no EPR signal was detected with only ortho-quinone. Cyclic voltammograms (CV) of $\boldsymbol{o}$-Q showed a reduction peak at $-0.63 \mathrm{~V}(v s \mathrm{Ag} / \mathrm{AgCl})$, and this was shifted to $0.65 \mathrm{~V}$ ( $v s \mathrm{Ag} / \mathrm{AgCl})$ in the presence of $\mathrm{La}(\mathrm{OTf})_{3}$, a positive shift as large as $0.95 \mathrm{~V}$ (Fig. 3c). All the other REE metals also showed large but varied positive shift of the reduction potential of $\boldsymbol{o}-\mathbf{Q}$ (Fig. S3). Hence, the oxidation capability of quinone was significantly enhanced by coordination to lanthanides ${ }^{19}$, facilitating single electron oxidation of iodide (TBAI) $\left(E_{\mathrm{ox}}=0.39 \mathrm{~V} v s \mathrm{Ag} / \mathrm{AgCl}\right)$. In comparison, the redox potential gap between free $\boldsymbol{o}-\mathbf{Q}$ and TBAI was $1.03 \mathrm{~V}$, largely disfavored for electron transfer ${ }^{28}$, and we did not observe any obvious change when mixing only $\boldsymbol{o}-\mathbf{Q}$ and TBAI. Next, reductive initiators other than iodide have been tested and the addition of cobaltocene or tetrathiafulvalene could also initiate the stochiometric reaction with comparable activity (Fig. 3d, Condition III and IV). 
Furthermore, the oxidation could also proceed when equal amount of $\boldsymbol{o}-\mathbf{Q}$ and hydroquinone $\boldsymbol{o}$ Qred were employed, such a combination was known to be able to generate semiquinone species under basic conditions and this was verified by UV-vis in our case ${ }^{25}$ (Fig. 3a and d, Condition V). Moreover, adding benzyl alcohol into the dark green solution of $\mathrm{LaI}_{3} /(\boldsymbol{o}-\mathbf{Q})_{2}$ showed gradually decrease of adsorption as monitored by UV-visible spectroscopy (Fig. 3a) and increasingly formation of benzaldehyde was observed by GC (Fig. S5). Similar quenching of the EPR signal was also clearly noted (Fig. 3b), indicating the consumption of the semiquinone intermediate. Taken together, these results strongly supported the involvement of the reductively generated semiquinone anionic radical in the active catalytic cycle and iodide served as a single electron donor for reductive generation of the active species.

It was noted that the reductively generated catalytic system in the absence of iodine species didn't cycle well under aerobic conditions (Fig. 3e). In contrast, the addition of catalytic amount of $\mathrm{TBAI}_{3}$ or $\mathrm{I}_{2}$ could reactivate the reaction (Fig. 3e). This observation implicated the direct regeneration of active catalytic species by oxygen was rather slow and iodine species may promote the re-oxidation of quinone catalyst ${ }^{29}$. To verify this hypothesis, the oxidation of catechol $\boldsymbol{o}$-Q $\mathbf{Q}_{\text {red }}$ was investigated. Though $\mathrm{TBAI}_{3}$ only was ineffective, its presence together with $\mathrm{La}(\mathrm{OTf})_{3}$ could significantly accelerate the re-oxidation (Fig. 3e), verifying the additional role of iodine species in recycling of reduced catalyst. We also examined the oxidation of hydroquinone $\boldsymbol{o}$-Qred under basic conditions. A quick appearance of green color, characteristic of semiquinone radical as detected by UV-vis (Fig. S6), was observed, which gradually changed to an orange color typical of $\boldsymbol{o}-\mathbf{Q}$ (Fig. S7). Conducting the same reaction under argon with $\mathrm{TBAI}_{3}$ as an oxidant also led to the formation of semiquinone radical (Fig. S8). In both cases, no green color was observed in the absence of lanthanum salt (Fig. S9), indicating the critical role of lanthanum metal in stabilizing 
semiquinone anionic radical. Mechanistically, $\mathrm{I}_{3}$ anion in concert with lanthanum metal may perform as a mediator to facilitate electron and proton transfer between catechol and oxygen. Their joint force not only enables the generation of the catalytically active semiquinone radical but also promotes re-oxidation of hydroquinones. 

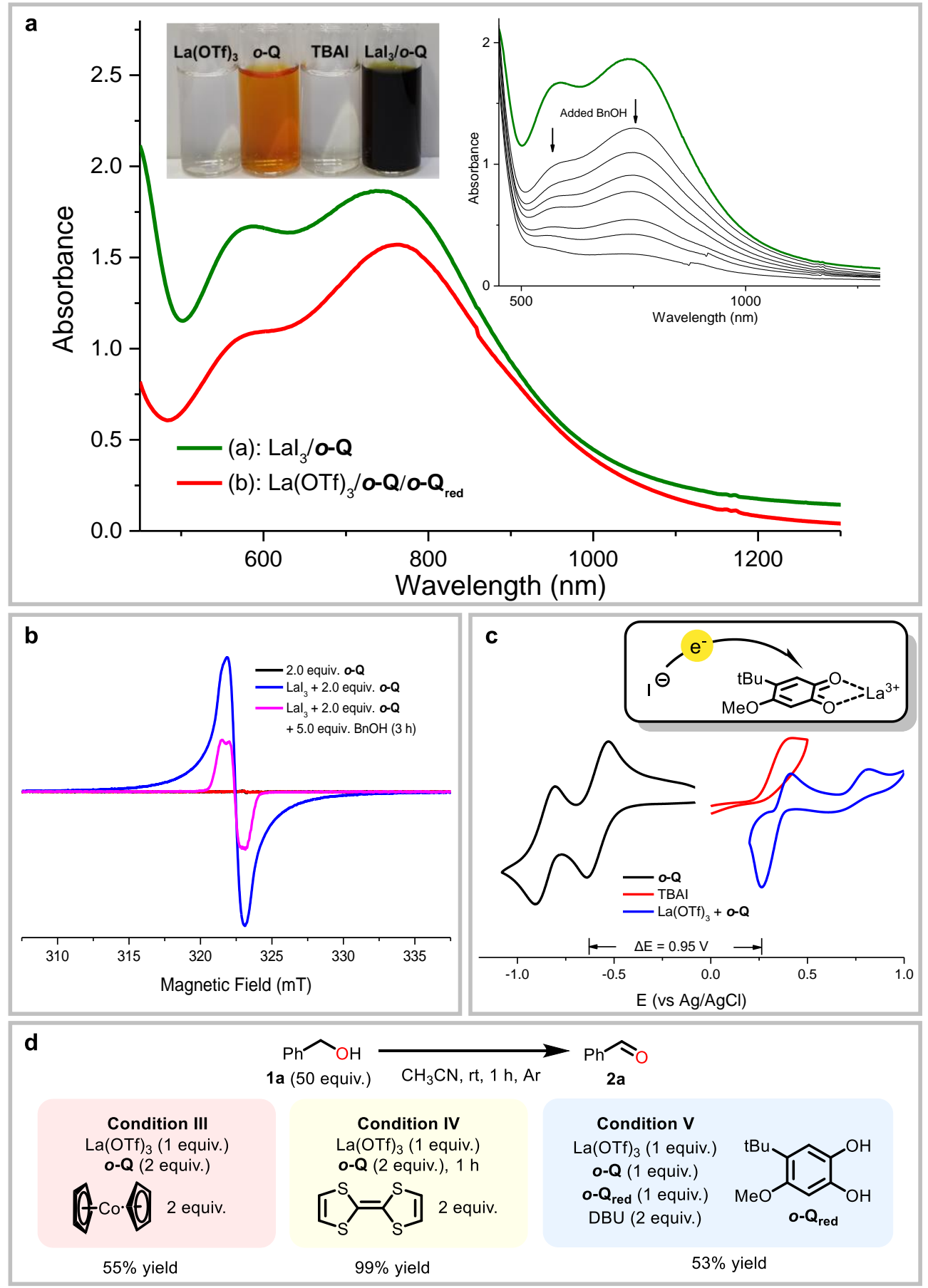

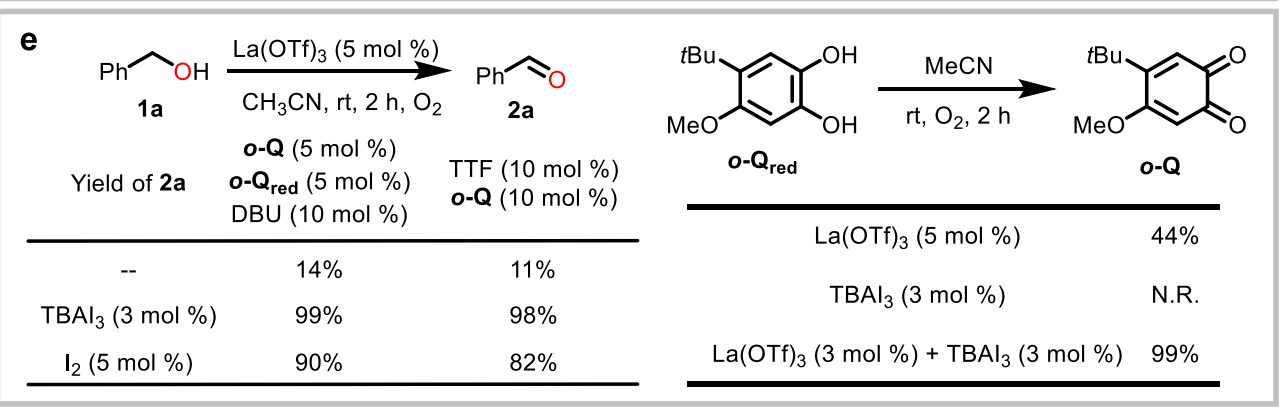


Figure 3. Reaction mechanism details. (a) UV-Vis spectrum. Sample concentration: $1.0 \mathrm{mM}$ in MeCN. a: $\mathrm{LaI}_{3}(1.0 \mathrm{mM})$ and $\boldsymbol{o}-\mathbf{Q}(2.0 \mathrm{mM})$; b: La(OTf) 3 (1.0 mM), o-Q (1.0 mM), o-Qred (1.0 $\mathrm{mM})$, DBU (2.0 mM). Inset: The time profile of stoichiometric benzyl alcohol quenching experiment. (Fig. S6) (b) EPR spectrum. Sample concentration: $\mathrm{LaI}_{3}(0.1 \mathrm{M})$ and $\boldsymbol{o}-\mathbf{Q}(0.1 \mathrm{M})$ in $\mathrm{MeCN}$ at $298 \mathrm{~K}$. (c) CV test. Sample concentration: $4.0 \mathrm{mM}$ in electrolyte solution (0.1 M $\mathrm{nBu}_{4} \mathrm{NPF}_{6}$ in $\mathrm{MeCN}$ ). (d) Other in-situ generated semiquinone species control experiments. TTF $=$ tetrathiafulvalene. Yields were based on the amount of $\mathrm{La}^{3+}$. (e) Role of iodine.

Based on the above experimental observations, a catalytic cycle was proposed as shown in Fig. 4. After coordinating with $\mathrm{LaI}_{3}, \boldsymbol{o}-\mathbf{Q}$ would be reduced by iodide anion via SET to form a radical dianion complex (Int-1) involving a La(III) metal center coordinated with at least two molecules of semiquinone radical anion $\mathbf{S Q}^{-}$. Alcohol would then coordinate with lanthanum center, following by oxidative dehydrogenation to afford the corresponding aldehyde or ketone. Ester and lactone could be formed via second dehydrogenation from a semi-acetal intermediate. The reduced hydroquinone $\mathbf{H Q}^{-}$complex (Int-2) would be re-oxidized by oxygen to regenerate Int-1 (Fig. 4). In Fukuzumi and Schelter's biomimic quinone catalysis, organic base was essential to facilitate the oxidative process and the reactions were believed to proceed via a step-wise deprotonation and hydride transfer mechanism, and the latter step may follow either an addition-elimination or direct hydride-transfer pathway. In our case, no obvious base effect was observed (Table S6) and significant KIE effect was found on both $\mathrm{O}-\mathrm{H}$ and $\mathrm{C}-\mathrm{H}$ of benzylic alcohol $\left(\mathrm{KIE}_{\mathrm{O}-\mathrm{H}}=1.40\right.$, KIE $\mathrm{C}_{-}$ $\mathrm{H}=2.19$ ) (Fig. S9). These observations were supportive of a coupled (concerted) O-H/C-H hydrogen transfers instead of stepwise deprotonation and hydride transfer. Each of the two semiquinone moieties $\mathbf{S Q}^{-}$may concurrently accept a hydrogen (Fig. 4). A Hammett plot with para-substituted benzyl alcohols revealed a preference for electron-rich substitutions with $\rho=$ - 
of hydride transfer. At this point, the detailed H-transfer mechanism remains to be elucidated, pending further structural characterization of the active intermediate (e.g. Int-1 or Int-2). We proposed a coupled proton-hydride transfer pathway to account for the experimental observations. Concerted $\mathrm{H}$-atom transfer, though can not be completely excluded, was unlikely considering the rather low BDE of hydroquinone $\boldsymbol{o}$-Qred $(75 \mathrm{kcal} / \mathrm{mol}$ by DFT vs $96 \mathrm{kcal} / \mathrm{mol}$ for $\mathrm{C}-\mathrm{H}$ bond in methanol). The absence of radical-rearrangement products with radical-probe substrates (e.g. 2i, $\mathbf{3 c}$ and $\mathbf{4 f a}$ ) disproved the existence of discrete radical intermediates during hydrogen-transfer, and is in support of hydride transfer. Preliminary DFT calculation were also carried out with a model complex. In the key transition state, an intramolecular $\mathrm{O}-\mathrm{H} \cdots \mathrm{O}$ hydrogen- bond would assistant C-H bond cleavage, which supported our coupled-proton-hydride transfer model (Fig. S15). The requirement of an open shell semiquinone anionic radical species could be understood by its ability in shuttling both proton and electron as well as its enhanced coordination capability toward lanthanum metal. In this regard, the SET activation opens a unique mechanistic scenario wherein two semiquinone anionic radicals are juxtaposed via lanthanum coordination for cooperative hydrogen transfer. 


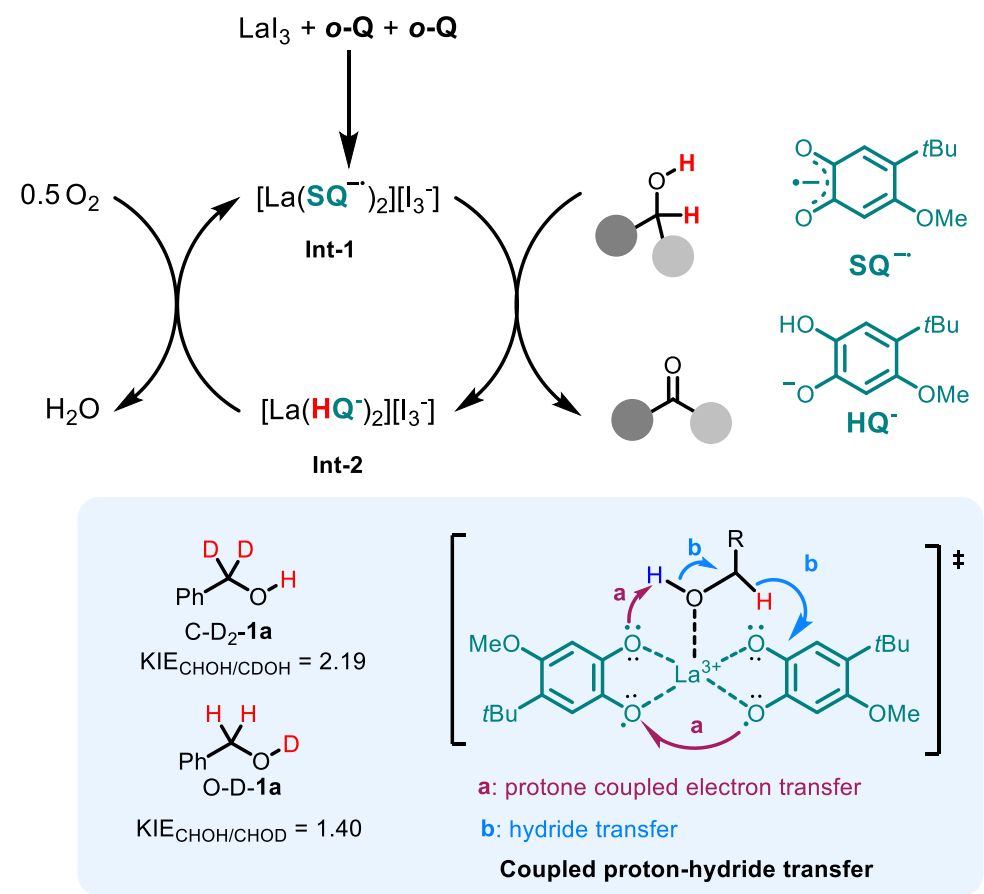

Figure 4. Proposed catalytic cycle (Upper). Lower: kinetic isotope effects of benzyl alcohol 1a (left) and a proposed coupled-proton-hydride transfer model (right).

The substrate scope was then explored under the optimal reaction conditions. The reactions worked well with para-substituted benzyl alcohols bearing either electron-donating (Fig. 5, 2a, 2b and $\mathbf{2 i}$ ) or electron-withdrawing substituents $(\mathbf{2 c})$. The reaction also tolerated functional groups such as thioether (2e), amine (2f), boronic acid ester (2g) and free phenols (2d and $2 \mathbf{h})$, to note that free phenols didn't work well in the Cu/TEMPO catalysis system ${ }^{30}$. Heterocyclic aromatic alcohols such as ferrocene methanol (2m) and furfuryl alcohol (2n) and allylic alcohols (2j-1) could be equally applied in the current catalysis. The reactions with aliphatic alcohols have also been examined to give a mixture of aldehyde and ester (from self-condensation). When the reaction was conducted in methanol, a sole formation of methyl ester could be achieved. Selected examples including long-chain alkyl (3a and 3b), cyclopropyl- (3c), bulky alkyl (3d and $\mathbf{3 e}$ ) and piperidinyl (3f and $\mathbf{3 g}$ ) were listed in Fig. 5, showing moderate to good activity. Diols such as 1,4-butanediol, 1,5-pentanediol and 1,6-hexanediol could be converted into the corresponding lactones (3h-k) with 
moderate to high yields. The catalysis also worked extremely well with secondary alcohols including aromatic (4aa-af), allylic alcohols such as verbenol and carveol (4c-e), acyclic (4h-i) and cyclic secondary alcohols (4fa-gb). The alcohols oxidation with testosterone (4m), androsterone (4n) and estradiol derivates (4oa-oc) went well under this catalysis. Large scale oxidations were also conducted and more than 200 turnover numbers could be achieved in one hour for all the three types of substrates.
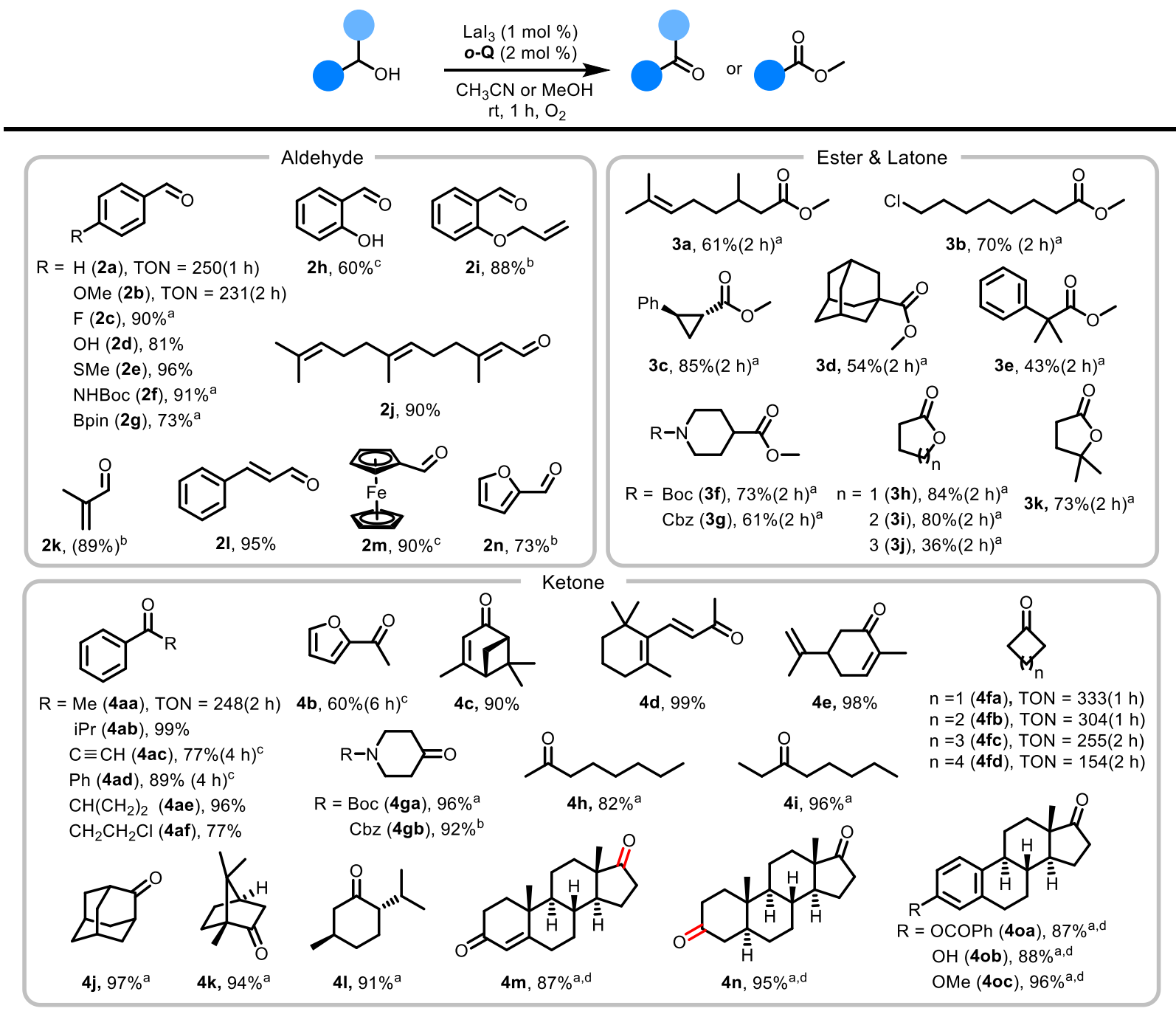

Figure 5. Substrate scope. Unless otherwise noted, reactions were conducted on a $1 \mathrm{mmol}$ scale with $1 \mathrm{~mL} \mathrm{MeCN}$ (for aldehyde, lactone and ketone) or $\mathrm{MeOH}$ (for ester) at room temperature under 1 atm $\mathrm{O}_{2}$, and yields were determined by flash column chromatography. Yields in 
parentheses were determined by ${ }^{1} \mathrm{H}$ NMR. ${ }^{\mathrm{a}} \mathrm{LaI}_{3}(2 \mathrm{~mol} \%), \boldsymbol{o}-\mathbf{Q}(4 \mathrm{~mol} \%) .{ }^{b} \mathrm{LaI}_{3}(4 \mathrm{~mol} \%), \boldsymbol{o}$-Q $(8 \mathrm{~mol} \%) .{ }^{c} \mathrm{La}(\mathrm{OTf})_{3}(5 \mathrm{~mol} \%), \boldsymbol{o}-\mathbf{Q}(10 \mathrm{~mol} \%), \mathrm{nBu} 4 \mathrm{NI}(15 \mathrm{~mol} \%)$ for $2 \mathrm{~h} .{ }^{\mathrm{d}} \mathrm{MeCN} / \mathrm{DCM}(\mathrm{v} / \mathrm{v}$ $=1: 1,0.5 \mathrm{M})$.

Though semiquinone radicals are frequently observed in quinoproteins, their function remains obscure. This study implies a possible functioning mode of semiquinone radicals beyond simply positing as a recycling intermediate of quinone cofactors. From the synthetic point of view, the reductive activation strategy as well as the resulted radical anion as redox ligand provides a new twist in exploring aerobic oxidation catalysis.

\section{Method}

General procedure for alcohol oxidation. A flame-dried $10 \mathrm{~mL}$ flask was flushed with $\mathrm{O}_{2}$ and equipped with an $\mathrm{O}_{2}$ balloon. $\mathrm{LaI}_{3}(5.19 \mathrm{mg}, 0.01 \mathrm{mmol})$ were added to the solution of $\boldsymbol{o}$-Q $\mathbf{Q}(3.88$ $\mathrm{mg}, 0.02 \mathrm{mmol})$ and alcohol $(1.0 \mathrm{mmol})$ in $1.0 \mathrm{~mL}$ of $\mathrm{CH}_{3} \mathrm{CN}$. The reaction was stirred at room temperature for $1 \mathrm{~h}$. After the reaction was completed, the crude reaction product was purified though a silica gel using 1:10-1:5 EtOAc/petro ether to give a pure product. For some volatile aldehyde, yields were determined by ${ }^{1} \mathrm{H}$ NMR.

\section{Data availability}

All other data that support the findings of this study are available within the article and its study are available from the corresponding author on reasonable request

\section{References}


1. Cheisson, T. \& Schelter, E. J. Rare earth elements: Mendeleev's bane, modern marvels. Science 363, 489-493 (2019).

2. Hibi Y. et al. Molecular structure of $\mathrm{La}^{3+}$-induced methanol dehydrogenase-like protein in methylobacterium radiotolerans. J. Biosci. Bioeng. 111, 547-549 (2011).

3. Cotruvo, J. A. The chemistry of lanthanides in biology: recent discoveries, emerging principles, and technological applications. ACS Cent. Sci. 5, 1496-1506 (2019).

4. Matsushita, K., Toyama, H., Yamada, M. \& Adachi, O. Quinoproteins: structure, function, and biotechnological applications. Appl. Microbiol. Biotechnol. 58, 13-22 (2002).

5. Prejano, M., Marino, T. \& Russo, N. How can methanol dehydrogenase from methylacidiphilum fumariolicum work with the alien $\mathrm{Ce}(\mathrm{III})$ ion in the active center? a theoretical study. Chem. -Eur. J. 23, 8652-8657 (2017).

6. Zhang, X., Reddy, S. Y. \& Bruice, T. C. Mechanism of methanol oxidation by quinoprotein methanol dehydrogenase. Proc. Natl. Acad. Sci. USA 104, 745-749 (2007).

7. Peterson, K. P. \& Larock, R. C. Palladium-catalyzed oxidation of primary and secondary allylic and benzylic alcohols. J. Org. Chem. 63, 3185-3189 (1998).

8. Brink, G.-J., Arends, I. W. C. E. \& Sheldon, R. A. Green, catalytic oxidation of alcohols in water. Science 287, 1636-1639 (2000).

9. Schultz, M. J., Park, C. C. \& Sigman, M. S. A convenient palladium-catalyzed aerobic oxidation of alcohols at room temperature. Chem. Comm. 2002, 3034-3035 (2002).

10. Jensen, D. R., Schultz, M. J., Mueller, J. A. \& Sigman, M. S. A well-defined complex for palladium-catalyzed aerobic oxidation of alcohols: design, synthesis, and mechanistic considerations. Angew. Chem. Int. Ed. 42, 3810-3813 (2003). 
11. Semmelhack, M. F., Schmid, C. R., Cortes, D. A. \& Chou, C. S. Oxidation of alcohols to aldehydes with oxygen and cupric ion, mediated by nitrosonium ion. J. Am. Chem. Soc. 106, 3374-3376 (1984).

12. Kumpulainen, E. T. \& Koskinen, A. M. Catalytic activity dependency on catalyst components in aerobic copper-TEMPO oxidation. Chem. -Eur. J. 15, 10901-10911 (2009).

13. Steves, J. E. \& Stahl, S. S. Copper(I)/ABNO-catalyzed aerobic alcohol oxidation: alleviating steric and electronic constraints of Cu/TEMPO catalyst systems. J. Am. Chem. Soc. 135, 15742-15745 (2013).

14. Badalyan, A. \& Stahl, S. S. Cooperative electrocatalytic alcohol oxidation with electronproton-transfer mediators. Nature, 535, 406-410 (2016).

15. Ma, S. et al. Development of a general and practical iron nitrate/TEMPO-catalyzed aerobic oxidation of alcohols to aldehydes/ketones: catalysis with table salt. Adv. Synth. Catal. 353, 1005-1017 (2011).

16. Itoh, S., Mure, M. \& Ohshiro, Y. Oxidation of D-glucose by coenzyme PQQ: 1,2-enediolates as substrates for PQQ oxidation. J. Chem. Soc., Chem. Commun. 1987, 1580-1581 (1987).

17. Itoh, S., Kawakami, H. \& Fukuzumi, S. Model studies on calcium-containing quinoprotein alcohol dehydrogenases. catalytic role of $\mathrm{Ca}^{2+}$ for the oxidation of alcohols by coenzyme PQQ (4,5-dihydro-4,5-dioxo-1H-pyrrolo[2,3-f]quinoline-2,7,9-tricarboxylic acid). Biochemistry 37, 6562-6571 (1998).

18. Itoh, S., Kawakami, H. \& Fukuzumi, S. Modeling of the chemistry of quinoprotein methanol dehydrogenase. oxidation of methanol by calcium complex of coenzyme PQQ via addition-elimination mechanism. J. Am. Chem. Soc. 119, 439-440 (1997). 
19. McSkimming, A., Cheisson, T., Carroll, P. J. \& Schelter, E. J. Functional synthetic model for the lanthanide-dependent quinoid alcohol dehydrogenase active site. J. Am. Chem. Soc. 140, $1223-1226$ (2018).

20. Janes, S. M. et al. A new redox cofactor in eukaryotic enzymes: 6-hydroxydopa at the active site of bovine serum amine oxidase. Science 248, 981-987 (1990).

21. Qin, Y., Zhang, L., Lv, J., Luo, S. \& Cheng, J.-P. Bioinspired organocatalytic aerobic C-H oxidation of amines with an ortho-quinone catalyst. Org. Lett., 17, 1469-1472 (2015).

22. Zhang, R., Qin, Y., Zhang, L. \& Luo, S. Mechanistic studies on bioinspired aerobic C-H oxidation of amines with an ortho-quinone catalyst. J. Org. Chem. 84, 2542-2555 (2019).

23. Largeron, M. Aerobic catalytic systems inspired by copper amine oxidases: recent developments and synthetic applications. Org. Biomol. Chem. 15, 4722-4730 (2017).

24. Yuasa, J. \& Fukuzumi, S. Thermochromism of the disproportionation equilibrium of $\pi$-dimer radical anion complexes bridged by scandium ions. Org. Biomol. Chem. 2, 642-644 (2004).

25. Ulas, G., Lemmin, T., Wu, Y., Gassner, G. T. \& DeGrado, W. F. Designed metalloprotein stabilizes a semiquinone radical. Nature Chem. 8, 354-359 (2016).

26. Bogart, J. A., Lewis, A. J. \& Schelter, E. J. DFT study of the active site of the XoxF-type natural, cerium-dependent methanol dehydrogenase enzyme. Chem. -Eur. J. 21, 1743-1748 (2015).

27. Witwicki, M. \& Jezierska, J. DFT insight into $o$-semiquinone radicals and $\mathrm{Ca}^{2+}$ ion interaction: structure, $g$ tensor, and stability. Theor. Chem. Acc. 132, 1383 (2013). 
28. Cheng, J.-P., Lu, Y., Zhu, X. \& Mu, L. Energetics of multistep versus one-step hydride transfer reactions of reduced nicotinamide adenine dinucleotide (NADH) models with organic cations and p-quinones. J. Org. Chem. 63, 6108-6114 (1998).

29. Wendlandt, A. E. \& Stahl, S. S. Bioinspired aerobic oxidation of secondary amines and nitrogen heterocycles with a bifunctional quinone catalyst. J. Am. Chem. Soc. 136, 506-512 (2014).

30. Hoover, J. M. \& Stahl, S. S. Highly practical copper(I)/TEMPO catalyst system for chemoselective aerobic oxidation of primary alcohols. J. Am. Chem. Soc. 133, 16901-16910 (2011).

\section{Acknowledgments}

We thank Prof. Jin-Pei Cheng and Prof. Lei Jiao for helpful discussions. This work is supported by the Natural Science Foundation of China (21672217, 21861132003 and 22031006) and Tsinghua University Initiative Scientific Research Program for financial support. S.L. is supported by the National Program of Top-notch Young Professionals.

\section{Author contributions}

S.L. conceived and directed the project. R.Z. optimized the reaction conditions, examined the substrate scope and studied the mechanism with help of L.Z. and M.-T.Z. L.Z. carried out DFT calculation. R.Z. and S.L. wrote the manuscript with contributions from all authors.

\section{Competing interests}

A patent application based on this chemistry has been submitted.

\section{Additional information}


Supplementary information is available for this paper at https://

Correspondence and requests for materials should be addressed to S.L.

Reprints and permissions information is available at www.nature.com/reprints.

Publisher's note Springer Nature remains neutral with regard to jurisdictional claims in

published maps and institutional affiliations. 